EPJ Web of Conferences 71, 00146 (2014)

DOI: $10.1051 /$ epjconf/20147100146

(C) Owned by the authors, published by EDP Sciences, 2014

\title{
Concrete Quarks - The Beginning of the End
}

\author{
George Zweig',a \\ ${ }^{1}$ MIT, Research Laboratory of Electronics, 26-169, 77 Massachusetts Ave, Cambridge, MA 02139
}

\begin{abstract}
A short history of the physics of strongly interacting particles is presented. Events leading to the discovery, and eventual acceptance, of concrete quarks are described. ${ }^{\mathrm{b}}$
\end{abstract}

\section{Introduction}

This year is the fiftieth anniversary of the discovery of quarks, and last year was the fortieth anniversary of the birth of QCD (Quantum Chromodynamics). QCD developed in two phases, the first involving the discovery of quarks, the second specifying the nature of their interactions. These phases arose from two very different traditions, that of Rutherford and Bohr, and that of Einstein. The first was grounded in observation, and the startling interpretation of what was observed, the second in a triumph of the imagination made possible by the power of quantum field theory. Here the first phase, culminating in the discovery and acceptance of concrete quarks, is described.

The story of QCD begins with the accidental discovery of spontaneous radioactivity by Henri Becquerel in 1896 who thought that X-rays, discovered just a few months earlier, might be emitted by phosphorescent substances. He noticed that crystalline crusts of uranium salts created silhouettes of great intensity when left in a drawer next to a photographic plate wrapped in black paper, even though the salt had not been exposed to sunlight (Fig. 1).

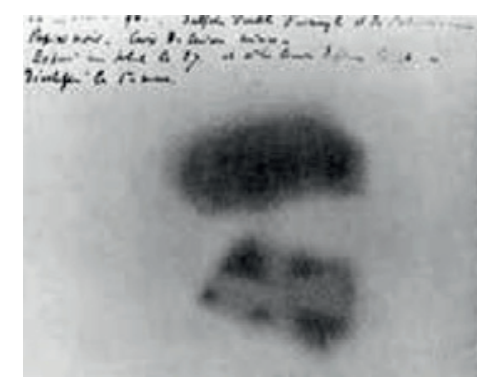

Figure 1. One of Becquerel's photographic plates fogged by exposure to radiation from potassium uranyl sulfate. The shadow of a metal Maltese Cross placed between the plate and the uranium salt is visible.

The nature of radioactivity was elucidated three years later by Ernest Rutherford who found that two types of particles, distinguished by their penetration power, were present in uranium radiation,

\footnotetext{
a e-mail: zweig@mit.edu

${ }^{b}$ Other aspects of quark history are given in Refs. [21] and [22]
}

This is an Open Access article distributed under the terms of the Creative Commons Attribution License 2.0, which permits unrestricted use, distribution, and reproduction in any medium, provided the original work is properly cited. 


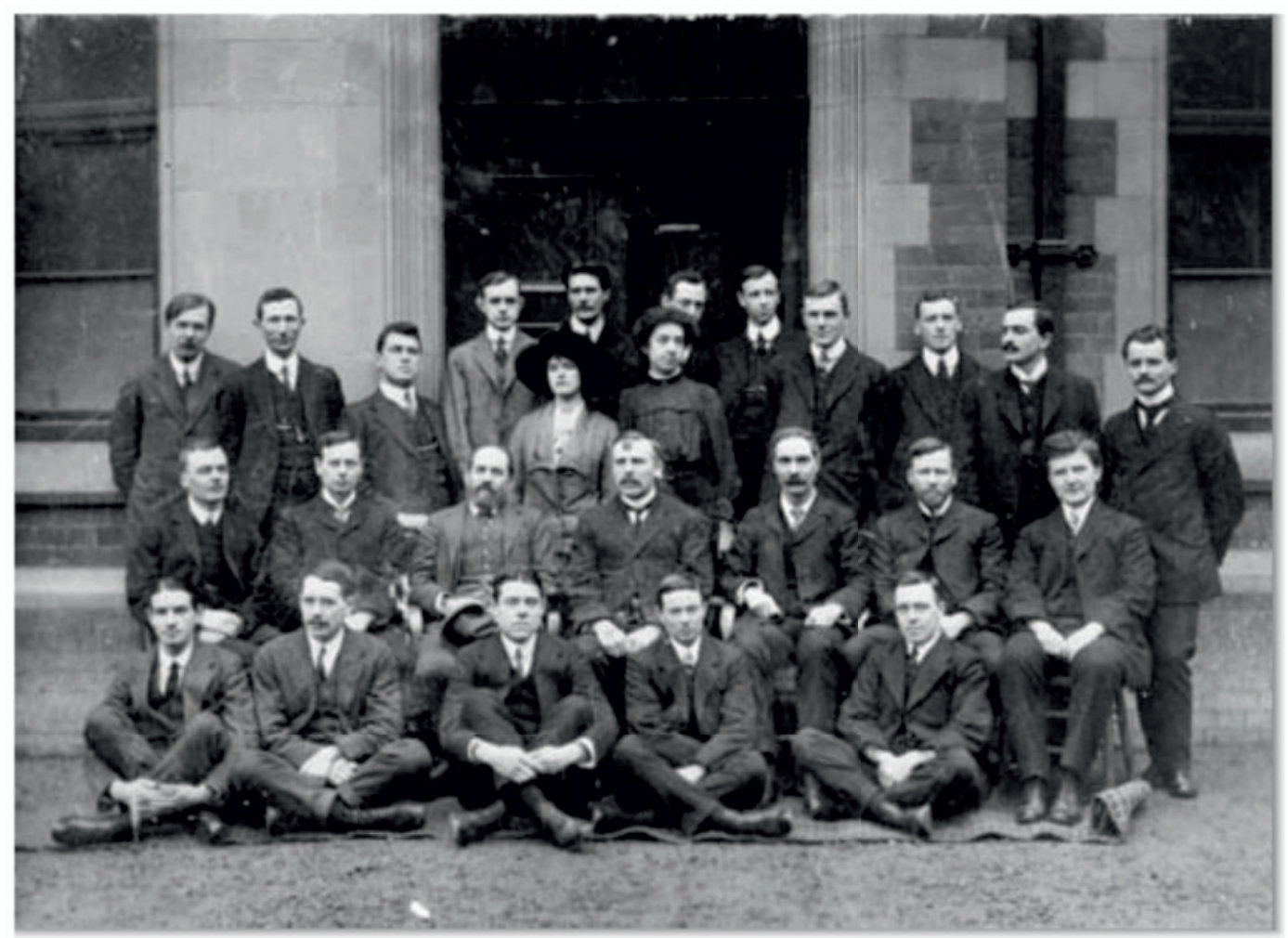

Figure 2. Rutherford's group at Manchester University in 1910. Rutherford is seated second row, center.

particles he called $\alpha$ and $\beta$. After three more years, Rutherford, with his young collaborator Frederick Soddy, interpreted the phenomena of radioactivity as "the spontaneous disintegration of [a] radio-element, whereby a part of the original atom was violently ejected as a radiant particle, and the remainder formed a totally new kind of atom with distinct chemical and physical character." Rutherford received the Nobel prize in Chemistry in 1908. Soddy also received a Nobel prize, but in 1921 for other work. ${ }^{1}$ A photo of Rutherford with his group at Manchester University, taken two years after his prize, is shown in Fig. 2.

QCD speaks of protons, neutrons, and more remotely, the nuclei of atoms. Thompson's discovery of the electron in 1897 indicated that the atom was divisible. The charge on an electron in a neutral atom must be cancelled by a positive charge. The first indication that this positive charge is point-like was reported by Hans Geiger and Ernest Marsden in 1909 [5]. Geiger was a postdoctoral fellow from Germany who came to Rutherford's lab to study the deflection of $\alpha$-particles as they passed through thin metal plates. Marsden was a 20 year old undergraduate from New Zealand, Rutherford's own country of origin. Geiger and Marsden reported that

\footnotetext{
${ }^{1}$ All told, eleven of Rutherford's students, collaborators, and members of his laboratory went on to receive the Nobel prize in Physics or Chemistry. Many more made remarkable but less recognized contributions. Rafi Muhammad Chaudhry went on to pioneer experimental nuclear physics in Pakistan, and with his student Mustafa Yar Khan, founded Pakistan's successful nuclear weapons program.
} 
"conclusive evidence was found of the existence of a diffuse reflection of the $\alpha$-particles. A small fraction of the $\alpha$-particles falling upon a metal plate have their directions changed to such an extent that they emerge again at the side of incidence."

This innocuous sounding statement, when properly interpreted, was revolutionary, completely contradicting Newton's laws of mechanics, and Maxwell's equations of electricity and magnetism as they had been formulated some 50 years earlier. Two years later in 1911 Rutherford published the proper interpretation [16], which negated the common view that an atom consisted of negatively charged electrons embedded within a ball of positive charge. He showed that a large point-like concentration of charge was essential to account for the dramatic reflection of the positively charged $\alpha$-particles back towards their source. ${ }^{2}$ This meant that positive and negative charges were separated in an atom, but how? Not statically, for they would fall together. Not circling one another, for they would radiate energy like tiny antennae spiraling together. Rutherford had discovered the nucleus, an object that could not exist according to the laws of physics.

When Marsden in 1914 and 1915 presented the first experimental evidence that nuclei contain protons, a second contradiction appeared. Why didn't the positively charged nucleus explode? What was binding protons together?

Shortly after receiving his Doctor's degree in 1911, Niels Bohr visited Rutherford's lab for several months, eventually settling there from 1914 to 1916 . During his stay Bohr correctly combined two incomprehensible ideas, that of charge separation in the atom, and that of Plank's quantization of radiation, into one incomprehensible idea, that electrons exist in "stationary states" within the atom, emitting quanta of light as they jumped between states. This view of the atom solved none of the contradictions with classical physics, but provided a conceptual framework within which the frequencies of spectral lines could be fruitfully organized, and their patterns contemplated.

The contradictions present in the Bohr atom were resolved with the introduction of quantum mechanics a decade later, which was formulated for atoms by Heisenberg solely in terms of the possible frequencies of light emitted as electrons changed their stationary states. His formulation consisted both of an equation involving a matrix of these possible frequencies, and a philosophy of how theoretical physics should be practiced. The contradictions inherent in Bohr's formulation of atomic physics would have been avoided if the equations of physics had been formulated entirely in terms of observables. When experimental particle physics came to consist primarily of two-particle collisions, the observables were scattering amplitudes, like the observables in the Geiger-Marsden experiment. In three papers during World War II Heisenberg argued that scattering amplitudes should also be organized into a matrix he called the S-matrix, and that the fundamental laws governing particles and their strong interactions should be formulated solely in terms of this matrix. Field theory, whose constructs and interactions were difficult or impossible to observe, was suspect. S-matrix theory in the form of the bootstrap [1] became the dominant school of thought in particle physics in the early 1960s.

My first exposure to nuclear physics came at the age of 10 in 1947, two years after the atomic bomb had been dropped on Hiroshima. One of my favorite after-school radio programs was the "Lone Ranger," sponsored by Kix breakfast cereal. Quite unexpectedly during a commercial break, the announcer asked his little listeners to send away for an "Atomic Bomb Ring" (Fig. 3). After mailing in my name, address, 15 cents, and a Kix box top, I received the ring, took it into a dark closet full of winter coats, and waited till my eyes had adapted to the dark. Removing the red cap and peering along the long axis of the "bomb," I was rewarded with brilliant punctate flashes of light as one $\alpha$-particle after another, emitted from a tiny piece of radioactive polonium, barreled into a zinc

\footnotetext{
${ }^{2}$ The charge could have either sign, was roughly proportional to the atomic number, and estimated to be $\pm 100 e$ for gold.
} 


\section{Lone Ranger Atom Bomb Ring Spinthariscope (1947 - early 1950s)}

This ring spinthariscope was known as the Lone Ranger Atom Bomb Ring and advertised as a "seething scientific creation." The Lone Ranger was more closely associated with silver bullets than atomic bombs but that's what it was called. When the red base (which served as a "secret message compartment") was taken off, and after a suitable period of time for dark adaptation, you could look through a small plastic lens at scintillations caused by polonium alpha particles striking a zinc sulfide screen.
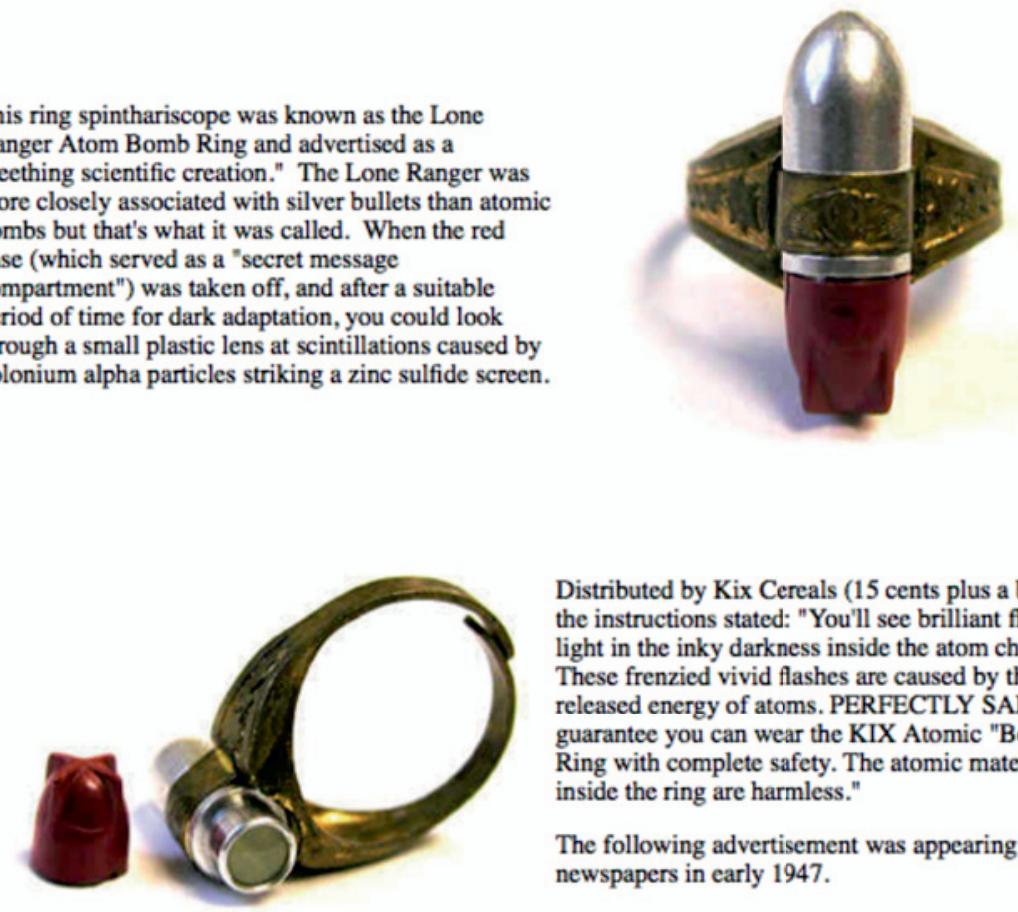

Distributed by Kix Cereals ( 15 cents plus a boxtop), the instructions stated: "You'll see brilliant flashes of light in the inky darkness inside the atom chamber. These frenzied vivid flashes are caused by the released energy of atoms. PERFECTLY SAFE - We guarantee you can wear the KIX Atomic "Bomb" Ring with complete safety. The atomic materials inside the ring are harmless."

The following advertisement was appearing in newspapers in early 1947.

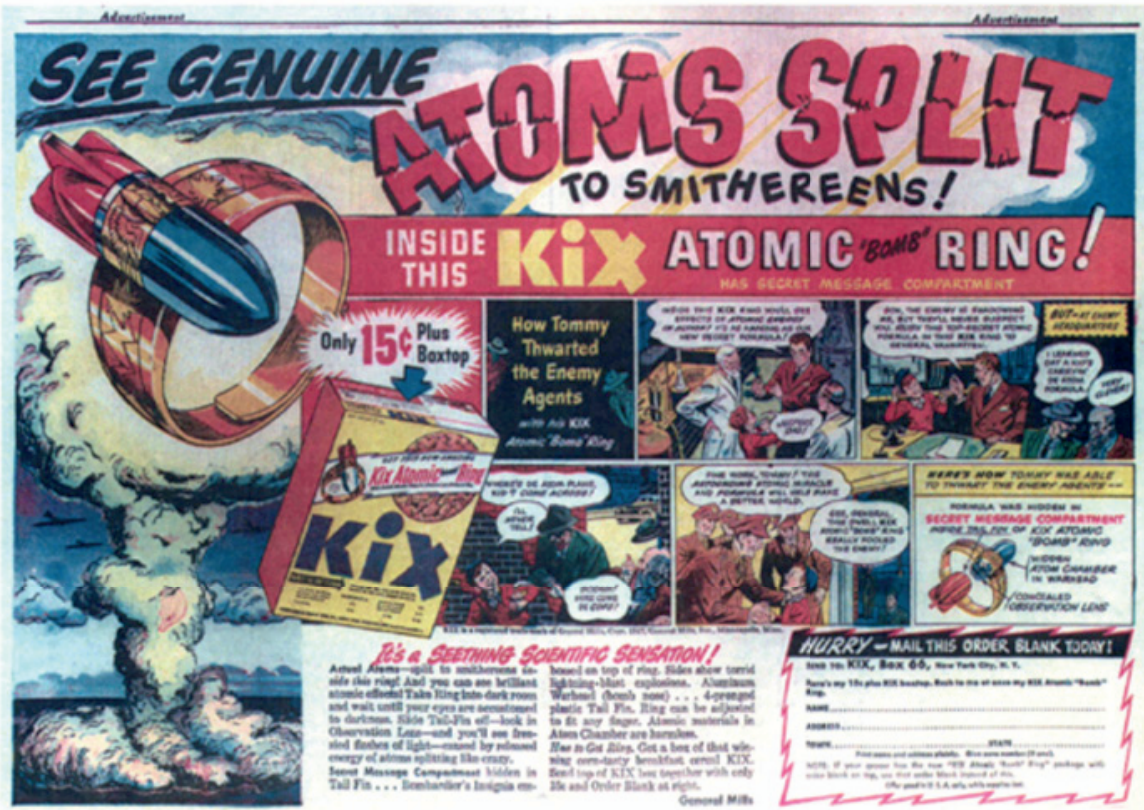

Figure 3. The Atom Bomb Ring based on Crookes's 1903 spinthariscope. 
sulfide screen (a spinthariscope invented by William Crookes in 1903). ${ }^{3}$ This was the same kind of screen used by Geiger and Marsden in their $1909 \alpha$-particle scattering experiment. ${ }^{4}$

That same year of 1947, C. F. Powell discovered the pion in emulsions. Earlier in 1932 J. Chadwick had discovered the neutron. With these discoveries the cast of characters within the nucleus was complete. Both Chadwick and Powell had been students of Rutherford.

But also in 1947, a strange form of matter was discovered. G. D. Rochester and C. C. Butler published two cloud chamber photographs of cosmic-ray events, providing the first evidence of the existence of the $K$ meson. Unlike the pion, whose existence had been predicted by Yukawa in 1935 to provide the force necessary to bind protons and neutrons together, the appearance of the $K$ meson was entirely unexpected. As I.I. Rabi famously quipped when the muon was discovered, "Who ordered that?"5

This proliferation of elementary particles led E. Fermi and C. N. Yang to publish a paper in 1949 titled "Are Mesons Elementary Particles?" with the abstract [4]:

"The hypothesis that $\pi$-mesons may be composite particles formed by the association of a nucleon with an antinucleon is discussed. From an extremely crude discussion of the model it appears that such a meson in most respects would have properties similar to those of the meson of the Yukawa theory."

However, in the body of the paper they caution,

"Unfortunately we have not succeeded in working out a satisfactory relativistically invariant theory of nucleons among which such attractive forces act."

This unsurmountable problem was to haunt Sakata's 1956 extension [15] of their work. He augmented the proton and neutron with the newly discovered $\Lambda$ to construct $K$ mesons out of pairs like the $\Lambda$ and antineutron $(\Lambda \bar{n})$, but the binding mechanism was still obscure. Creating the $\pi$ and $K$ would also be problematic for the bootstrap [1], the fulfillment of Heisenberg's S matrix theory. Advocates of the bootstrap ignored this problem.

By 1957 the list of "elementary particles" had grown to $19 .{ }^{6}$ M. Gell-Mann and A. Rosenfeld summarized the situation in a paper for the Annual Review of Nuclear Sciences [7]. Working with E.P. Rosenbaum, an editor for Scientific American, Gell-Mann reworked this material for the general public in an article titled "Elementary Particles" [6]. The 19 elementary particles they listed are shown in Fig. 4.

In the same issue of the Annual Review of Nuclear Science, S. Lindenbaum discussed a relatively new phenomenon, resonances in pion nucleon scattering, of which there were at least two (Fig. 5). Elementary particles and resonances coexisted side by side in journals and theoretician's minds, unconnected. Resonances, the "elephants in the room," were about to explode in number.

What distinguished resonances from the so-called elementary particles was their lifetimes. Resonances were created and decayed via the strong interactions. They lived for only $10^{-23}$ seconds,

\footnotetext{
${ }^{3}$ Crookes was remarkable. He also discovered thallium in 1861 , invented the radiometer, and developed the Crookes tube that was later used by W.C. Roentgen and J.J. Thomson in their discoveries of the X-ray and the electron. As a young teenager wandering the stacks in the Detroit Public Library, I was fascinated by a book written by Crookes chronicling seances held in his house in the 1870 s, not realizing his seminal contribution to the development of nuclear physics and the creation of the atomic bomb ring.

${ }^{4}$ I was so moved by the magic of the ring that I got a second one, not knowing that the half-life of Polonium 210 was only 138 days.

${ }^{5}$ We still don't know who ordered the muon.

${ }^{6}$ Willis Lamb, in the first paragraph of his 1955 Nobel Prize Lecture, joked that he had "heard it said that 'the finder of a new elementary particle used to be rewarded by a Nobel Prize, but such a discovery now ought to be punished by a $\$ 10,000$ fine.'"
} 
Point particles

\begin{tabular}{|l|l|}
\hline \multicolumn{2}{|c|}{ Spin 1/2 leptons } \\
\hline \hline Particle & Mass \\
\hline$e^{-}$ & 1 \\
$\mu^{-}$ & 206.7 \\
$\nu$ & 0 \\
\hline
\end{tabular}

\begin{tabular}{|l|l|}
\hline \multicolumn{2}{|c|}{ Spin 1 photon } \\
\hline \hline Particle & Mass \\
\hline$\gamma$ & 0 \\
\hline
\end{tabular}

Extended particles (strongly interacting)

\begin{tabular}{|l|l|l|}
\hline \multicolumn{3}{|c|}{ Spin 1/2 baryons } \\
\hline \hline Multiplet & Particle & Mass $\left(m_{e}\right)$ \\
\hline$\Xi$ & $\Xi^{0}$ & $?$ \\
& $\Xi^{-1}$ & 2585 \\
\hline \multirow{3}{*}{$\Sigma$} & $\Sigma^{-1}$ & 2341 \\
& $\Sigma^{+}$ & 2325 \\
& $\Sigma^{0}$ & 2324 \\
\hline$\Lambda$ & $\Lambda$ & 2182 \\
\hline \multirow{2}{*}{$\mathrm{N}$} & $\mathrm{n}$ & 1838.6 \\
& $\mathrm{p}$ & 1836.1 \\
\hline
\end{tabular}

\begin{tabular}{|l|l|l|}
\hline \multicolumn{3}{|c|}{ Spin 0 mesons } \\
\hline \hline Multiplet & Particle & Mass \\
\hline \multirow{4}{*}{$\pi$} & $\pi^{+}$ & 273.2 \\
& $\pi^{-1}$ & 273.2 \\
& $\pi^{0}$ & 264.2 \\
\hline \multirow{4}{*}{$\mathrm{K}$} & $\mathrm{K}^{+}$ & 966.5 \\
& $\mathrm{~K}^{-}$ & 966.5 \\
& $\mathrm{~K}_{1}^{0}$ & 965 \\
& $\mathrm{~K}_{2}^{0}$ & 965 \\
\hline
\end{tabular}

Figure 4. The elementary (long-lived) particles in 1957 [6]. 


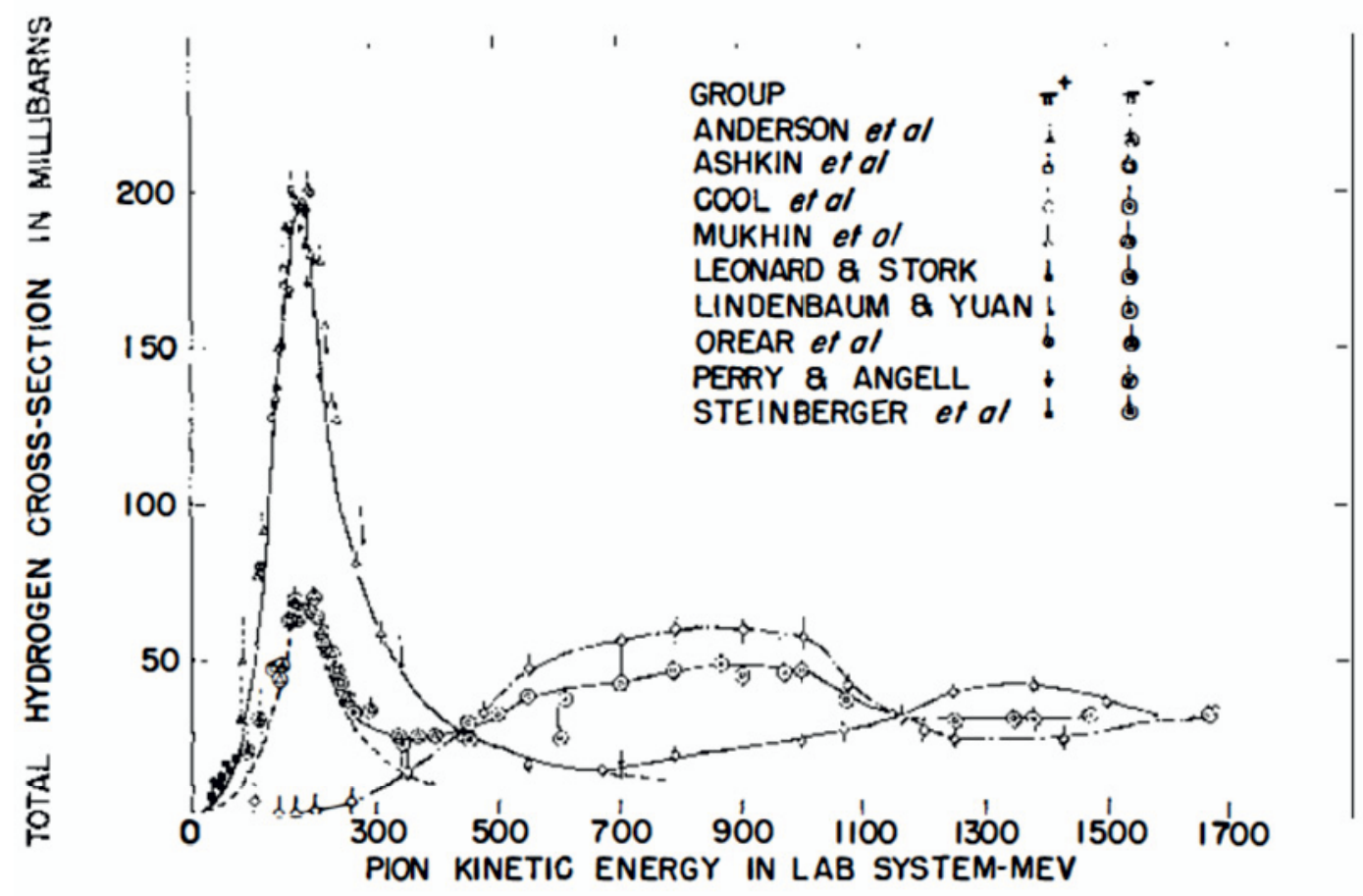

Figure 5. $\pi^{ \pm}+p$ total cross-sections as of 1957 showing evidence for the first pion-nucleon resonances [10].

about the time it takes light to travel across a proton. Elementary particles lived much longer, either being stable, or decaying slowly through the electromagnetic or weak interactions. Elementary particles were grouped into two classes, point-like and extended in size. Only later was it realized that the long-lived extended elementary particles and the short-lived resonances were "cut from the same cloth."

In the summer of 1957 I had just finished my sophomore year as a math major at the University of Michigan. I remember reading the Gell-Mann-Rosenbaum Scientific American article. My reaction can be summarized by lines from a Bob Dylan song of later years,

"But something is happening here

And you don't know what it is

Do you, Mister Jones?"7

\section{Caltech}

Two years later in 1959, 50 years after the Geiger- Marsden experiment, I started graduate school in physics at Caltech, and shortly thereafter started my $\mathrm{PhD}$ thesis as an experimentalist in Alvin Tollestrup's group, piggy backing on their proposed study of $K^{+} \rightarrow \pi^{+}+\pi^{0}+\gamma$, to measure the polarization of the $\mu$, out of the plane of decay, in $K_{\mu 3}$ decay. A non-zero value would imply a violation of time-reversal symmetry. This was the first "user's group" experiment at Caltech, involving

\footnotetext{
${ }^{7}$ Bob Dylan, Ballad of a Thin Man, final track on Side One of Highway 61 Revisited, 1965
} 
1 faculty member, 2 research fellows, and 2 graduate students. The experimental equipment was built and tested at Caltech over a two-year period, but run at the Bevatron in Berkeley. After 21 half-days of running in the early spring of 1962, an adventure that should be chronicled elsewhere, I returned to Caltech with several hundred thousand spark-chamber photographs. A preliminary scan found no effect. I then faced an additional two years of tedious analysis determining the magnitude of errors, and establishing an accurate upper bound. With this unpleasant prospect, I embraced denial and went camping in the Yucatan peninsula.

On returning at the end of summer, I switched to theory. Screwing up my courage, I asked Murray Gell-Mann if he would be my thesis advisor. He seemed the natural choice; he supervised many graduate students, all of whom graduated quickly, and I had spoken with him on several occasions after Alvin suggested that I talk to him about Alvin's $K$-decay experiment that was meant to elucidate the mysterious $\Delta I=1 / 2$ rule for weak nonleptonic decays. When asked, Murray immediately said "No," paused, and then said he was going to the East Coast on sabbatical, but would "talk to Dick." It wasn't until I returned from CERN two years later that I would speak with Murray once again.

"Dick" was Richard Feynman. I would never have had the courage to approach him. I had just watched him, in the second lecture of his gravity course, for the first time, write down the Feynman rules for gravity, and compute the scattering of Mercury off the sun, getting the advance of the perihelion in 45 minutes, a result that I had seen H.P. Robertson, the grand old man of general relativity, obtain only after seven months into his course on general relativity. ${ }^{8}$.

About a week later I asked Feynman if he would be my thesis advisor. He replied in a hoarse low-pitched voice, "Murray says your okay, so you must be okay." Then he laid down the ground rules. I was to see him every Thursday from 1:30 to 4:15 when we would adjourn for tea, and then proceed to the physics colloquium. Each week I prepared frantically for our Thursday sessions, trying to pick some subject that would interest him, never touching any subject more than once, not even my thesis. My job was to make sure that Feynman was never bored. During the 1962-63 academic year we covered essentially all of particle physics. An outline follows:

- Theory in the abstract:

- Axiomatic field theory was championed by Arthur Wightman at Princeton. Feynman hated it, and I didn't see how it helped explain the wealth of observations being made.

- Theory related to belief was championed by Geoffrey Chew at Berkeley. At a La Jolla conference in June 1961 in support of the bootstrap Chew said:

"I believe the conventional association of fields with strongly interacting particles to be empty. ... field theory..., like an old soldier, is destined not to die but just fade away."

- Theory related to experiment involving:

- Particle classification (no dynamics): The groups $\mathrm{G}_{2}$ and $\mathrm{SU}_{3}$ were in contention.

- Dynamics (no particle classification): Although the bootstrap had its problems, Fred Zachariasen was able to bootstrap the $\rho$ meson from two pions (Fig. 6). Forces between particles are created

\footnotetext{
${ }^{8}$ Robertson, of the "Robertson-Walker metric," had the distinction of rejecting a paper submitted to the Physical Review by Einstein. Einstein claimed that an accelerating mass would not emit gravitational radiation. Robertson's review was longer than Einstein's paper. Einstein was furious, but Robertson's evaluation was correct (http://physicstoday.org/journals/ doc/PHTOAD-ft/vol_58/iss_9/43_1.shtml?bypassSSO=1). Robertson was one of many luminaries in the Caltech Physics Department. Carl Anderson, chairman, discovered both the positron and muon, and his department included six soon-to-be Nobel Prize winners: R. Feynman, W. Fowler, M. Gell-Mann, Shelly Glashow, R. Mössbauer, and K. Wilson; Sidney Coleman and R. Dashen were students, and Y. Ne'eman and J.J. Sakurai visitors. If that wasn't enough, you could always go across campus and talk with ex-particle-physicist Max Delbrück, who had invented molecular biology, or Linus Pauling, a phenomenologist par excellence. I got the impression that it was possible to do things. Discoveries were happening, just down the hall!
} 
by the exchange of particles. If the $\rho$ exists, it will be exchanged between two pions, creating an attractive force strong enough to bind them into a state with the quantum numbers of the $\rho$. Equating the particle exchanged $(\rho)$ with the state it creates leads to a determination of the $\rho$ mass and $\rho \pi \pi$ coupling constant that have the right order of magnitude [17]. As simply described by Chew, Gell-Mann, and Rosenfeld, the bootstrap was the future [2].
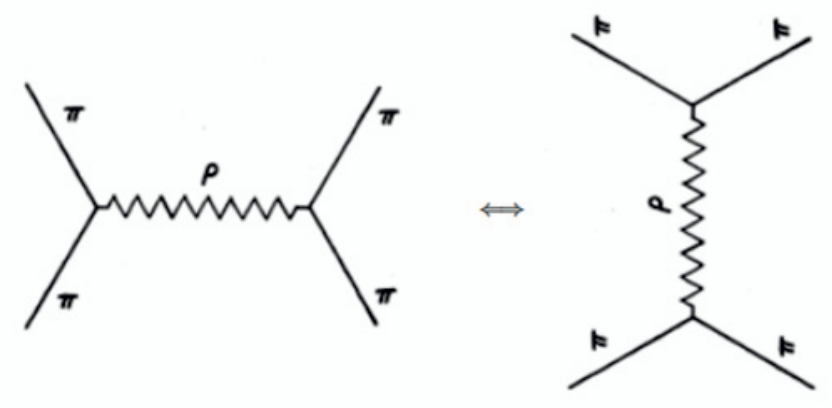

Figure 6. The $\rho$ bootstrap. Exchanging a $\rho$ binds two pions into a $\rho$ [17].

Feynman was sympathetic to the goals of S-matrix theory and the bootstrap, but never used it himself. I didn't see how to bootstrap the $\pi$ meson.

- Experimental physics:

- More elementary particles and many more resonances were discovered:

- Point particles: The 4th lepton $\left(v_{\mu}\right)$,

- Extended elementary particles: An 8th spin 0 meson $(\eta)$ and an 8 th spin $1 / 2$ baryon $\left(\Xi^{0}\right)$,

- Resonances: 26 meson resonances $\left(\rho, \omega, K^{*}, \cdots\right)$, the first of which had been discovered only 2 years earlier in 1961 .

\section{Something unbelievable}

One Thursday afternoon I showed Feynman a paper in Physical Review Letters titled "Existence and Properties of the $\phi$ Meson" [3]. Other papers had presented evidence for the existence of the $\phi$, but this paper found no evidence where evidence was primarily expected. It was the nonexistence of a decay mode that should have broadened the width of the $\phi$ that fascinated me. Fig. 7 shows the Dalitz plot for $K^{-}+p \rightarrow \Lambda+K+\bar{K}$. Note the long thin vertical pencil of $K \bar{K}$ events at the left edge of phase space clearly indicating the existence of the $\phi$. So far, so good.

But now look at Fig. 8 where the three pion mass distribution is displayed. There is no statistically significant evidence for a peak, so there is no evidence for the decay $\phi \rightarrow \pi^{+}+\pi^{-}+\pi^{0}$, which I thought should be dominant. This decay channel is expected to proceed through the chain of reactions $\phi \rightarrow \rho+\pi$, followed by $\rho \rightarrow \pi+\pi$. The authors comment on this unexpected absence:

"The observed rate [for $\phi \rightarrow \rho+\pi$ ] is lower than ... predicted values by one order of magnitude; however the above estimates are uncertain by at least this amount so that this discrepancy need not be disconcerting."

But I was very disconcerted. My calculations indicated that the $\rho \pi$ mode was suppressed by at least 


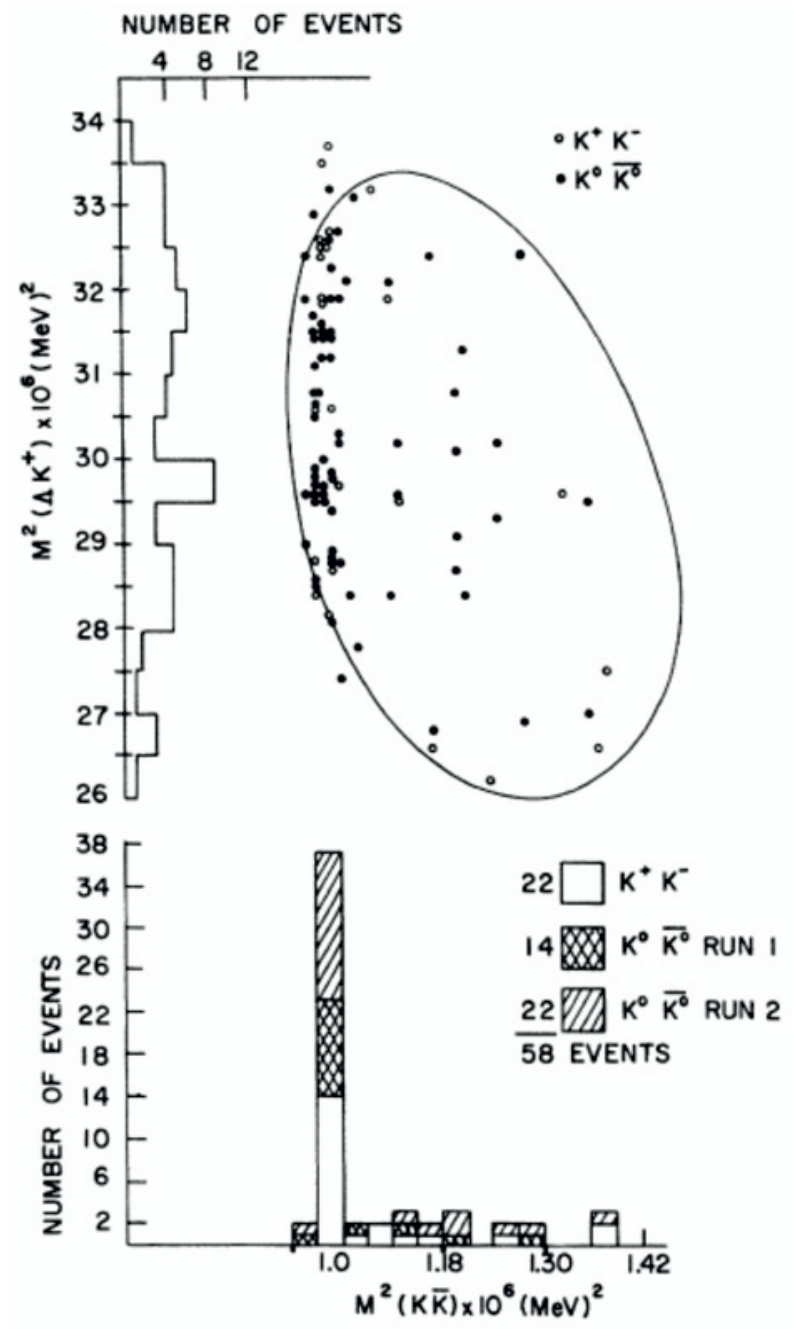

Figure 7. Fig. 1 from [3]. "Dalitz plot for the reaction $K^{-}+p \rightarrow \Lambda+K+\bar{K}$. The effective-mass distributions for $K \bar{K}$ and $\Lambda K^{+}$are projected on the abscissa and ordinate." The cluster of events at the edge of phase space indicate the existence of a meson called the $\phi$ decaying into $K+\bar{K}$.

two orders of magnitude, ${ }^{9}$ an unprecedented suppression for the strong interactions. I had learned from Feynman that in the strong interactions everything that can possibly happen does, and with

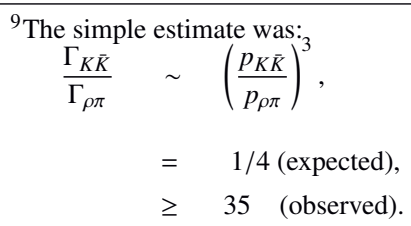

Here $p_{i j}$ is the momentum of either particle $i$ or $j$ in the rest frame of the $\phi$. 


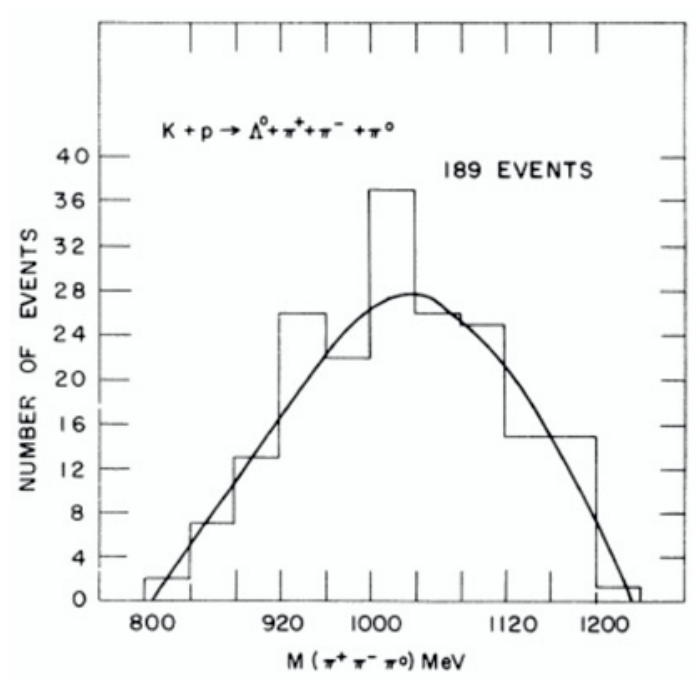

Figure 8. Fig. 4 from [3]. "The $M\left(\pi^{+} \pi^{-} \pi^{0}\right)$ distribution from the reaction $K^{-}+p \rightarrow \Lambda+\pi^{+}+\pi^{-}+\pi^{0}$." The absence of a large peak above phase space (solid line) indicates the suppression of the decay $\phi \rightarrow \rho+\pi$.

the maximum strength allowed by unitarity. Suppression implied symmetry, but no symmetry was present. Therefore the suppression must be dynamical, but no dynamical mechanism was available.

Feynman was not disconcerted. He launched into a tirade about how unreliable experiments were, and explained that at the time he proposed the $\mathrm{V}-\mathrm{A}$ theory for the weak interactions all experiments were against him, and those experiments were wrong. ${ }^{10}$

\section{The explanation}

I couldn't stop thinking about the suppression of $\phi$ decay, and finally realized that this paradox could be resolved by assuming that hadrons had constituents that obeyed a simple dynamical rule when they decayed [19, 20]. Like Fermi and Yang, and later Sakata, I assumed that mesons were constructed out of fermion-antifermion pairs, but the fundamental fermions were not nucleons, but new fields called "aces," for reasons that will become apparent (they are now associated with constituent quarks, but unlike constituent quarks, aces are also the fundamental fields in electromagnetic and weak currents). With this assumption, the problems Fermi and Yang had in making the pion a nucleon-antinucleon bound state disappeared. Some unknown force, not the nuclear force, bound aces together. As in the Sakata model, the constituents consisted of an isotopic spin doublet $N_{0} \equiv\left\{p_{0}, n_{0}\right\}$ analogous to the nucleons $\{p, n\}$, and an isotopic spin singlet $\Lambda_{0}$ analogous to the $\Lambda$, together with their antiparticles $\left\{\bar{p}_{0}, \bar{n}_{0}, \bar{\Lambda}_{0}\right\}$. The vector mesons with their constituents are shown in Fig. 12 d. The sawtooth line connecting an ace $a$ (shaded circle) with an antiace $\bar{a}$ (open circle) represents the spring holding them

\footnotetext{
${ }^{10}$ The V-A theory (1957) was initially at variance with angular correlations measured in $\mathrm{He}^{6}$ decay and the absence of the decay $\pi^{-} \rightarrow e^{-}+\bar{v}$, which was not seen by S. Lokanathan and J. Steinberger in 1955. In 1958, in CERN's first major discovery, Fazzini, Fidecaro, Merrison, Paul and Tollestrup observed this decay at the predicted rate, confirming V-A (also proposed by Marshak and Sudarshan, who led Gell-Mann to the same conclusion). As a testimony to the difficulty of measurement, Steinberger was an outstanding experimentalist, a student of Fermi, who later shared the Nobel Prize for demonstrating that the electron and muon each have their own neutrinos.
} 


\section{EPJ Web of Conferences}

AN $\mathrm{SU}_{3}$ MODEL FOR STRONG IMTERACTION SYMETRRY AND ITS BREAKIITG

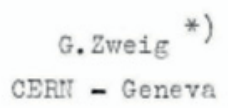

Both mesons and baryons are constructed from a set of three fundamental particles called aces. The aces break up into an isospin doublet and singlet. Each ace carries baryon number $\frac{1}{3}$ and is consequently fractionally charged. $\mathrm{SU}_{3}$ (but not the Eightfold Way) is adopted as a higher symmetry for the strong interactions. The breaking of this symetry is assumed to be universal,boing due to mass differences among the aces. Bxtensive space-time and group theoretic structure is then predicted for both mesons and baryons, in agreement with existing experimental information. $\mathrm{h}$ experimental search for the aces is suggested.

*) This work was supported by the Air Porce office of Scientific Research and the National Academy of Sciences - National Research Council, U.S.A.

8182/TH. 401

17 January 1964

Figure 9. Title page of Ref. [19]. Only certain $\mathrm{SU}_{3}$ representations, quantum numbers, and decays are allowed, constraints not found in the Eightfold Way. 
together. The $a \bar{a}$ pair has angular momentum $L=0$, and total spin $S=\frac{1}{2}+\frac{1}{2}=1$. The pion, a pseudoscalar meson, has the same constituents as the $\rho$, but the spins of its constituents cancel, making $S=0$. A meson decays when its $a \bar{a}$ constituents fly off in different directions. Their separation induces a polarization of the vacuum creating an $\bar{a}^{\prime} a^{\prime}$ pair that also splits, forming $a \bar{a}^{\prime}$ and $a^{\prime} \bar{a}$, the decay products (Fig. 10). With this rule of ace conservation (a component of Zweig's

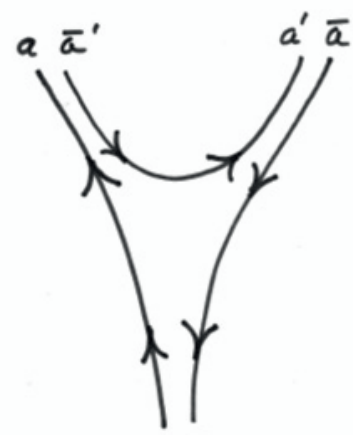

Figure 10. The decay of meson $a \bar{a}, a$ is an ace, $\bar{a}$ an antiace.

rule), aces in the initial state must appear in the final state, i.e., "aces don't eat each other." Since the $\phi$ consists only of $\Lambda_{0}$ and $\bar{\Lambda}_{0}$, constituents not found in $\rho$ or $\pi$, the decay $\phi$ to $\rho+\pi$ can not take place, and empirically is strongly suppressed. ${ }^{11}$ Remarkably, the constituent assignment for the mesons required to suppress $\phi \rightarrow \rho+\pi$ gave mass formulae for the vector mesons that worked exceptionally well (see below). ${ }^{12}$

In order to obtain the observed baryon spectrum, it was necessary to assign aces baryon number $B=1 / 3$. This distinguished them from the $\{p, n, \Lambda\}$ of the Sakata model, which failed to group the low-lying baryons into an $\mathrm{SU}_{3}$ octet representation (Fig. 4). The charge $Q$ of an ace was then given by the Gell-Mann Nishijima mass formula $Q=e\left[I_{z}+(B+S) / 2\right]$ (here, and only here, $S$ is strangeness).

These ideas were extended to characterize additional properties of hadrons and their strong, electromagnetic, and weak interactions. The essence of the model as it appeared in two CERN preprints at the start of 1964, (Fig. 9, [19], and [20]), is as follows: ${ }^{13}$

1. Hadrons have point fermion constituents with baryon number $1 / 3$,

2. There is a correspondence between leptons, the point particles of the weak interactions, and aces, the point particles of the strong interactions. In 1963 four leptons were known, hence the name aces. ${ }^{14}$ This correspondence was just a hunch, in the Einstein tradition.

3. The ultimate number of constituents was unknown. Representing them by $\bullet, \diamond, \diamond$ and $\diamond$ was not general enough. To allow for the possibility of an expanding set, each constituent was

\footnotetext{
${ }^{11}$ Originally a graphically different, but functionally equivalent, visual representation of the decay was given, involving a "tinker toy" construction (see Fig. 13 of Ref. [20]).

${ }^{12}$ Okubo obtained the suppression of $\phi$ decay, and mass formulae, with a different argument that didn't involve constituents[12].

${ }^{13}$ Work on aces was almost finished by Thanksgiving 1963 when Ricardo Gomez, a Caltech Research Fellow I worked with on the $K$-decay experiment at Berkeley, came to visit. When I told him what I was doing, he smiled and said I was "crazy." He wanted to go to the Bataclan, the one and only sexy cabaret in town (Calvin's Geneva). Much to my surprise, we skipped a very long line and were seated at a small table up front with a free bottle of champagne. The manager thought Ricardo was Ricardo Gomez, the famous bicycle racer, and Ricardo did nothing to disabuse him of this idea.

${ }^{14}$ With some clairvoyance, I would have called them dice.
} 
represented by a regular polygon of ever increasing size, corresponding to ever increasing mass, in the series circle $=p_{0}$, triangle $=n_{0}$, and square $=\Lambda_{0}$. The fourth ace, when found, would be represented by a pentagon,

4. Each hadron is represented by a linear combination of $a \bar{a}^{\prime}$ pairs (deuces) for mesons, and $a a^{\prime} a^{\prime \prime}$ triplets (treys) for baryons. The deuces or treys are weighted by $\mathrm{SU}_{3}$ coefficients to form meson and baryon wave functions, e.g., $\rho^{0}=\frac{1}{\sqrt{2}}\left(p_{0} \bar{p}_{0}-n_{0} \bar{n}_{0}\right)$, indicating that the $\rho^{0}$ is equally likely to be a $p_{0} \bar{p}_{0}$ or $n_{0} \bar{n}_{0}$, neglecting electromagnetic interactions.

Aces provide a rationale for the existence of $\mathrm{SU}_{3}$ symmetry, but more restrictively, lead to unique predictions for the existence of only certain $\mathrm{SU}_{3}$ representations and quantum numbers, and provide relations among masses, and coupling constants. Baryons only come in groups of 1,8 , and $10(3 \times 3 \times 3=1+8+8+10)$, mesons only in groups of 1 and $8(3 \times \overline{3}=1+8)$, with these two $\mathrm{SU}_{3}$ representations mixing strongly for vector mesons, leading to a group of 9 . Keeping track of ace spins, and allowing aces to have angular momentum, connects the spin-parity of baryons with the dimension of their $\mathrm{SU}_{3}$ representations, and creates higher mass excited states. Assuming aces in the lightest mesons and baryons have zero angular momentum leads to the observed baryon octet $\left(J^{P}=\frac{1}{2}^{+}\right)$, decuplet $\left(\frac{3}{2}^{+}\right)$, and singlet $\left(\frac{1}{2}^{-}\right)$, as well as a pseudoscalar meson octet $\left(J^{P C}=0^{-+}\right)$and vector meson nonet $\left(1^{+-}\right)$. Mesons with certain quantum numbers are forbidden, i.e., those with $J^{P C}=0^{--}$and those in the series $0^{+-}, 1^{-+}, 2^{+-}, \ldots$ Such "exotic" states may still be absent, but weakly bound deuteron-like bound states with exotic quantum numbers may now exist.

5. Aces, not hadrons, interact:

(a) For the strong interactions, hadrons decay when their constituents separate and initiate the formation of decay products (Zweig's rule, ${ }^{15}$ Figs. 10 and 11).

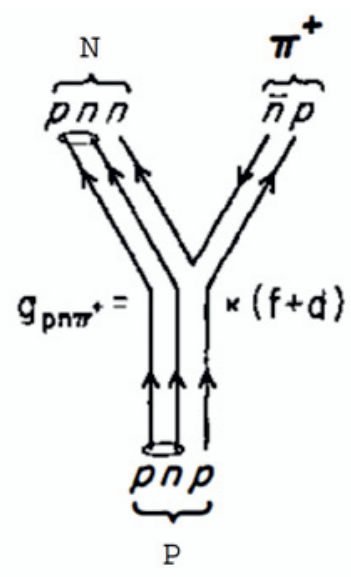

Figure 11. Graphical representation of the " $f+d$ " contribution to meson-baryon coupling. The "little loop" encloses anti-symmetrized aces. A different contribution to the coupling arises if the anti-symmetrized aces are separated in the decay, one ace terminating in the baryon, the other in the meson. The subscript " 0 " on aces is suppressed. Adapted from Ref. [11].

Graphically, when a meson decays into other mesons, it decays in all ways the deuces present can be connected, each connection having the same amplitude. The decay amplitude is proportional to the sum of these amplitudes, each amplitude weighted by the

\footnotetext{
${ }^{15}$ Gell-Mann enjoyed using Rosner's and Harari's term, the "twig rule" (twig is derived from the German word zweig, meaning "branch"). Zweig's rule differs from that of Okubo's or lizuki's because it not only says what is forbidden, but also what is allowed, and by how much. It involves enumerating, weighting, and summing all graphs available to a hadron for decay. An application of Zweig's rule that is not covered by the OZI rule is found in [20], and illustrated in Fig. 11.
} 
amplitude of the deuces in the meson wave functions (examples given in Figs. 10 and 11 of Ref. [20]). This is an application of Feynman's "sum over all paths" formulation of quantum mechanics.

(b) The electromagnetic or weak interactions of hadrons occur through the interaction of their constituents with the photon or intermediate vector boson. For example, when the neutron $n$ undergoes $\beta$-decay $\left(n \rightarrow p+e^{-}+v\right)$, it is really its $n_{0}$ constituent that $\beta$-decays $\left(n_{0} \rightarrow\right.$ $\left.p_{0}+e^{-}+v\right)$, the lighter $p_{0}$ becoming a constituent of the final-state proton. In this respect aces behave like fields in a field theory, i.e., like "current quarks [8]."

6. The mass of a hadron is the weighted average of the masses of its deuces or treys, the weights given by the relative probabilities of the hadron existing in its possible deuce or trey configurations. The mass of a deuce $D_{a}^{\bar{a}^{\prime}}$ or trey $T_{a a^{\prime} a^{\prime \prime}}$ is the sum of their constituent masses minus their pairwise binding energies. Subscripts refer to aces, superscripts to antiaces. There are no three-body forces. Ace mass differences are assumed to be greater than their binding energy differences, so symmetry breaking arises primarily from ace mass splittings $(|\Delta E|<<|\Delta m|)$ :

(a) For mesons: $m\left(D_{a}^{\bar{a}^{\prime}}\right)=m(a)+m\left(\bar{a}^{\prime}\right)-E_{a}^{\bar{a}^{\prime}}$. The binding energy $E_{a}^{\bar{a}^{\prime}}$ depends on the $\mathrm{SU}_{3}$ representation of the meson containing the deuce, and on the total spin $\vec{S}$ and angular momentum $\vec{L}$ of the $a \bar{a}$ system.

(b) For baryons: $m\left(T_{a a^{\prime} a^{\prime \prime}}\right)=m(a)+m\left(a^{\prime}\right)+m\left(a^{\prime \prime}\right)-E_{a a^{\prime} *}-E_{a * a^{\prime \prime}}-E_{* a^{\prime} a^{\prime \prime}}$. A trey $T_{a a^{\prime} a^{\prime \prime}}$ in a baryon is represented by a triangle with the aces $a, a^{\prime}$ and $a^{\prime \prime}$ at its vertices, with $E_{a a^{\prime} *}=E_{a^{\prime} a *}$. The binding energies depend on the $\mathrm{SU}_{3}$ representation of the baryon containing the trey, and on the spins and angular momentum of the aces.

The strong interaction symmetry $\mathrm{SU}_{3}$ is broken by distinguishing the mass and binding energies of $\Lambda_{0}$ from $N_{0}$, (Fig. 12).

The electromagnetic symmetry $\mathrm{SU}_{2}$ is broken by distinguishing the mass and binding energies of $n_{0}$ from $p_{0}$ (Fig. 13).

No potential function is assumed. This is not the naive quark model! The naive quark model missed the point. It made detailed assumptions about the potential that were surely incorrect, with very little to show for it. I had no idea how the potential varied with distance, just that it gave rise to a two-body force. ${ }^{16}$

\section{The question}

Would you, the reader, have adopted aces as constituents? To help answer this question, some results beyond $\mathrm{SU}_{3}$ symmetry from the 80 page CERN report are outlined below. Except when in curly brackets, the masses in $\mathrm{MeV}$ are those known at the time.

1. $\mathrm{SU}_{3}$ symmetry breaking: $m\left(p_{0}\right)=m\left(n_{0}\right)<m\left(\Lambda_{0}\right)$, leading to:

A hierarchy of baryon relations, e.g., $J^{P}=\frac{1}{2}^{+}$octet:

\footnotetext{
${ }^{16}$ When Finn Ravndal first came to Caltech as a graduate student in 1968 he was eager to work on the quark model, and was very surprised to find that Murray and I were not thinking about quarks. I had done what I could five years earlier, and didn't know how to find the force binding aces. Even if I guessed correctly, I thought the theory would be so nonlinear that I couldn't prove the guess correct. Murray wasn't trying to find the force because he didn't think quarks existed. Ken Young, one of Murray's brightest graduate students, laments that Murray didn't ask him to compute the force between two quarks when he asked Murray for a thesis problem in 1970, a problem that was assigned to Politzer and Wilczek by their thesis advisors two years later, after Gerard 't Hooft had found the answer, but didn't fully publish his result.
} 
a.
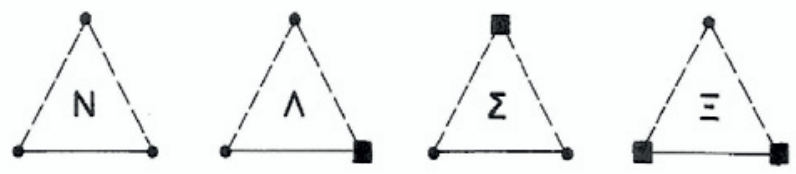

b.
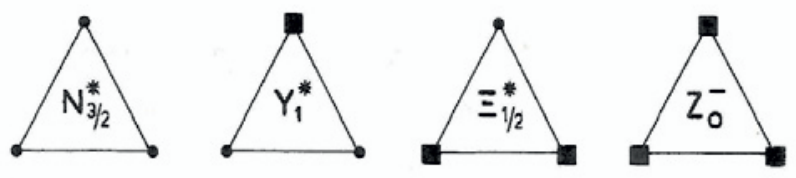

c.

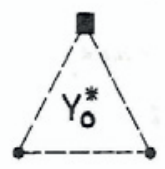

d.

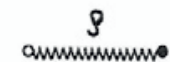

$\stackrel{\omega}{\text { annuminumio }}$

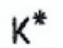

$\varphi$

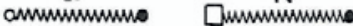

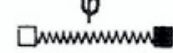

Figure 12. Fig. 2 of [19]. "We view the particle representations with unitary symmetry broken. One of the three aces has now become distinguishable from the other two. It is pictured as a shaded square. ... The mass splittings within representations are induced by making the squares heavier than the circles. Since the same set of aces are used to construct all hadrons, mass relations connecting mesons and baryons may be obtained." The assignment of constituents to the vector mesons was suggested by the observation that the $\rho$ and $\omega$ had the same mass, and the square of the $K^{*}$ mass was the average of the mass squares of the $\rho$ and $\phi$.

(a) All binding energies are the same, or $|\Delta E|<<|\Delta m|$. Then, the stranger a baryon in a multiplet, the heavier it is:

i. $m(N)<m(\Lambda) \approx m(\Sigma)<m(\Xi)$,

$9391115 \quad 1193 \quad 1315$

ii. $\frac{1}{2}[m(\Xi)+m(N)] \approx \frac{1}{2}[m(\Lambda)+m(\Sigma)]$.

$$
1127 \quad 1154
$$

(b) Averaging relations for the binding energies hold, i.e.,

$\frac{1}{2}\left(E_{\Lambda_{0} \Lambda_{0} .}+E_{\alpha \beta}\right) \approx E_{\Lambda_{0} \alpha .} \approx E_{\Lambda_{0} \beta}, \alpha, \beta=p_{0}, n_{0}:$

i. $\frac{1}{2}[m(\Xi)+m(N)] \approx \frac{1}{4}[3 m(\Lambda)+m(\Sigma)]$.

$$
1127 \quad 1134
$$

(Gell-Mann Okubo mass formula) 


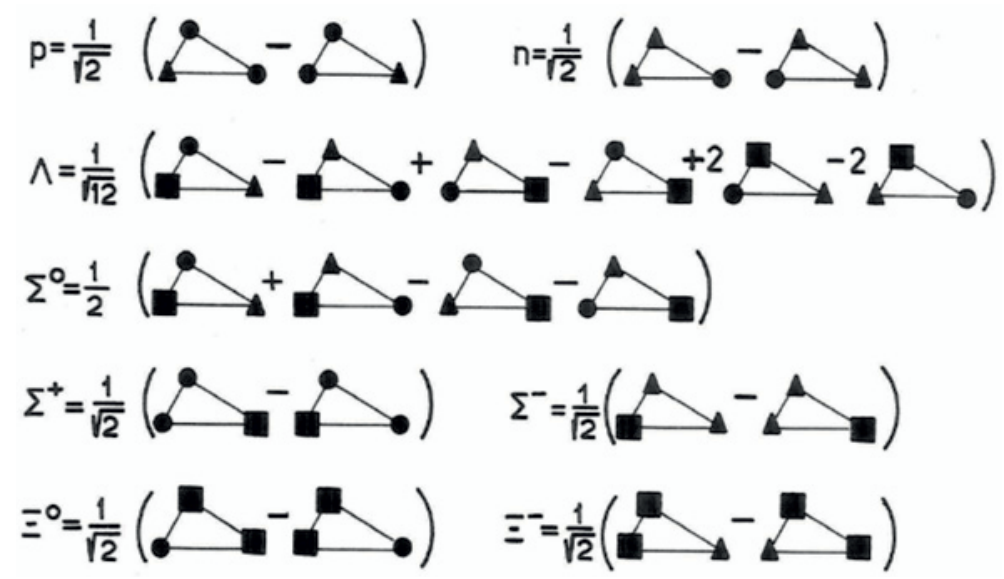

Figure 13. Fig. 3 of [20]. "After $\mathrm{SU}_{3}$ has been broken by the strong and electromagnetic interactions the baryon octet looks like this. The three aces are now completely distinguishable from one another." A baryon wave function is a linear combination of treys. The trey coefficients come from $\mathrm{SU}_{3}$. Although aces are point-like, the mass of an ace is monotonically related to the size of its symbol.

A hierarchy of meson relations, ${ }^{17}$ e.g., $J^{P C}=1^{--}$nonet:

(a) All binding energies are the same, or $|\Delta E|<<|\Delta m|$ :

i. $m^{2}(\rho) \approx m^{2}(\omega)<m^{2}\left(K^{*}\right)<m^{2}(\phi)$.
$750^{2} \quad 784^{2} \quad 888^{2} \quad 1018^{2}$

(b) Averaging relations for the binding energies hold, i.e., $\frac{1}{2}\left(E_{\Lambda_{0}}^{\bar{\Lambda}_{0}}+E_{\alpha}^{\bar{\beta}}\right) \approx E_{\Lambda_{0}}^{\bar{\beta}} \approx E_{\alpha}^{\bar{\Lambda}_{0}}, \alpha, \beta=p_{0}, n_{0}:$

i. $m^{2}(\phi) \approx 2 m^{2}\left(K^{*}\right)-m^{2}(\rho)$.

$1018^{2} \quad 1007^{2}$

2. SU(2) symmetry breaking: $m\left(p_{0}\right)<m\left(n_{0}\right)$, leading to:

A hierarchy of baryon relations, e.g., $J^{P}=\frac{1}{2}^{+}$octet:

(a) All binding energies are the same, or $|\Delta E|<<|\Delta m|$. Then, the more negative a baryon in a multiplet, the heavier it is:

i. $m(p)<m(n)$,

938939

\footnotetext{
${ }^{17}$ Mass relations were linear for baryons, and quadratic for mesons, in analogy with the linear Dirac equation for fermions, and the quadratic Klein-Gordon equation for mesons.
} 
ii. $m\left(\Sigma^{+}\right)<m\left(\Sigma^{0}\right)<m\left(\Sigma^{-}\right)$,

$11901193 \quad 1196$

iii. $m\left(\Xi^{0}\right)<m\left(\Xi^{-}\right)$.

$1315 \quad 1321$

(b) Averaging relations for the binding energies hold, i.e.,

$\frac{1}{2}\left(E_{p_{0} p_{0} .}+E_{n_{0} n_{0}}\right) \approx E_{p_{0} n_{0} .}, \cdots:$

i. $\frac{1}{2}\left[m\left(\Sigma^{+}\right)+m\left(\Sigma^{-}\right)\right] \approx m\left(\Sigma^{0}\right)$.

$1193.4 \pm 0.3 \quad 1193.2 \pm 0.7$

(c) All binding energies are different:

i. $m(n)-m(p)+m\left(\Xi^{-}\right)-m\left(\Xi^{0}\right)=m\left(\Sigma^{-}\right)-m\left(\Sigma^{+}\right)$.

$$
\begin{array}{ll}
7.3 \pm 1.3 & 8.3 \pm 0.5
\end{array}
$$

2013 masses: $\quad\{8.14 \pm 0.21\} \quad\{8.08 \pm 0.08\}$

2013 mass differences: $\quad\{0.06 \pm 0.22\}$

This remarkable relation currently still holds to the known accuracy of the mass differences.

3. Additional relations for the baryon decuplet and pseudoscalar meson octet.

4. Additional mesons with orbital excitations $(L=1,2, \cdots)$ and $\vec{L} \cdot \vec{S}$ mass splittings.

5. Cross multiplet relations:

(a) $m(N)<m(\Lambda) \Longrightarrow m^{2}(\rho)<m^{2}\left(K^{*}\right)$,

(b) $m\left(\Xi^{*}\right)-m\left(\Sigma^{*}\right) \approx m(\Xi)-m(\Sigma)$,

$145 \quad 122$

(c) $m^{2}\left(K^{*}\right)-m^{2}(\rho) \approx m^{2}(K)-m^{2}(\pi)$.

$$
0.22 \mathrm{Gev}^{2} \quad 0.22 \mathrm{Gev}^{2}
$$

Were these many successful relations a result of over-fitting? ${ }^{18}$ Probably not, because over the past 50 years the accuracy of these relations has either remained essentially the same, or improved.

\section{The difficulties}

All this was not as easy as it looks. Although aces resolved the problem of $\phi$ decay, explained many regularities, and made predictions that could easily be tested, some predictions contradicted experiment, and even violated basic theoretical principles.

Matt Roos's "definitive" compilation of resonances, published in the 1963 Reviews of Modern Physics, lists 26 meson resonances [14]. We now know that 19 of these resonances do not exist, and of those 19, 7 are exotic, i.e., cannot be made from deuces. As recounted previously [21]:

\footnotetext{
${ }^{18}$ Certain simple relations like $m(\Xi)=[3 m(\Sigma)-m(N)] / 2$, which were correct to electromagnetic mass splittings, were considered accidental.
} 


\begin{abstract}
"Matt Roos's ... compilation of particles and their properties referred to several hundred experimental papers. I read essentially all of them, taking care to understand how each measurement was made. Then an accurate appraisal of the results of each experiment was possible. Rational choices between conflicting experiments usually could be made. Training in experimental physics was helpful in this process."
\end{abstract}

In this way it was possible to separate wheat from chaff. Others did not have the interest, training, or patience.

A major theoretical problem was the existence of the celebrated $\Omega^{-}$with spin $S=\frac{3}{2}$ constructed from three identical aces all in the same state, thereby violating Pauli's spin-statistics theorem. I didn't have a resolution to this problem, but thought it eventually would be resolved, much like the contradictions with Rutherford's atom and Bohr's orbits were eventually resolved by quantum mechanics. ${ }^{19}$

Equally problematic, aces had no place in current dogma. They were incompatible with nuclear democracy, the basis of the bootstrap, and much prevailing thinking. In addition, since aces were not observed as free particles, formulating a theory of strong interactions in term of them was incompatible with Heisenberg's requirement that theory be based solely on observables. Like Copernicus's view of the solar system, simplicity was no excuse for challenging what was known to be true. ${ }^{20}$

Finally, working with aces made me uncomfortable. I knew what real theories looked like. As a student I had read and understood Schwinger's papers on QED. I had written "professional" papers [18]. Nothing I now did looked like that. Talking about aces in public was embarrassing, because I understood how theorists thought, and what many thought of me. ${ }^{21}$ In spite of this, a good part of me believed that aces were real particles, and that the beginnings of a solution to the proliferation problem of hadrons had finally been found. Aces were real particles because they had dynamics. They moved from one hadron to another, avoiding annihilation, either forwards or backwards in time. They spun and rotated around one another giving rise to $\vec{L} \cdot \vec{S}$ mass splittings. In addition, they interacted with light and underwent $\beta$ decay. I applied the "duck" test: If it walks like a duck, swims like a duck, and quacks like a duck, it's probably a duck. Others disagreed.

\title{
7 The reaction
}

After returning from CERN in the early fall of 1964, I went into Murray's office and told him all about aces. Sometimes Murray would close his eyes when someone talked to him, but this time his eyes stayed open. After I finished at the blackboard he exclaimed from behind his desk "Oh, the concrete quark model. That's for blockheads!" As late as five years after the deep inelastic scattering experiments at SLAC provided conclusive evidence for electrons scattering off point particles in nucleons, Murray still did not accept the existence of constituent quarks inside of hadrons [9]:

\footnotetext{
${ }^{19}$ Feynman's 1970 solution to the spin-statistics problem was to make quarks bosons! The abstract to a paper written by Feynman, Kislinger, and Ravndal titled "The $\Delta I=1 / 2$ Rule from the Symmetric Quark Model" reads "The $\Delta I=1 / 2$ rule for the weak non-leptonic hyperon decays will result from quark currents interacting at a point, if the quarks obey Bose statistics." The paper was withdrawn before publication when Feynman learned that this idea had been previously proposed (Ravndal, private communication).

${ }^{20}$ Wegener's theory of continental drift provides another example of discovery contradicting dogma. Evidence from geology and paleontology clearly showed that Africa and South America were connected sometime in the past, but this idea was not accepted because the Earth's crust was thought to be "frozen." A dynamical mechanism for driving continents apart was not yet known.

${ }^{21}$ Having worked at the Bevatron in the early 1960s I appreciated the excitement and beauty of Berkeley and the San Francisco area. I wanted a job at UC Berkeley. Gerson Goldhaber presented my application package at a physics faculty meeting, but a senior theorist blocked the appointment, passionately arguing that the ace model was the work of a "charlatan" (Goldhaber, private communication).
} 
"In these lectures I want to speak about at least two interpretations of the concept of quarks for hadrons and, the possible relations between them.

First I want to talk about quarks as 'constituent quarks'. These were used especially by G. Zweig (1964) [italics added] who referred to them as aces. ..."

It is more precise to say: "These were introduced by G. Zweig (1964)..." After all they did not exist as a tool in a toolbox for anyone to use.

Murray then goes on to say:

The whole idea is that hadrons act as if they are made up of quarks, but the quarks do not have to be real. ..."

That's a mischaracterization of my idea.

"There is a second use of quarks, as so-called 'current quarks' which is quite different from their use as constituent quarks ...

If quarks are only fictitious there are certain defects and virtues. The main defect would be that we never experimentally discover real ones and thus will never have a quarkonics industry. The virtue is that then there are no basic constituents for hadrons hadrons act as if they were made up of quarks but no quarks exist - and, therefore, there is no reason for a distinction between the quark and bootstrap picture: they can be just two different descriptions of the same system, like wave mechanics and matrix mechanics. [italics added]"

This was Murray's vision.

Although Feynman had no quarrel with "current quarks [8]," or the current-quark aspect of aces, he disliked Zweig's rule. Every time I would "explain" it to him he became angry, and said that it didn't make sense. Unitarity mixed all states with the same quantum numbers, so the suppression of the $\phi$ to $\rho \pi$ was not possible. He also believed that the correct theory of the strong interactions should not allow one to say which particles are elementary, a key element of the bootstrap.

\section{Acceptance}

Late in May of 1968 I bumped into Feynman as we both were walking to the Greasy (the Caltech cafeteria) for lunch. He was very excited about the High Energy Physics course he had just finished teaching, and was back in full swing after a lull in research following his unsuccessful attempt to renormalize gravity. After listing the many areas he had covered in his course he stopped, turned to me and asked, "Did I miss anything Zweig?" Patiently, once again, I told him about aces. This time he was quiet, intent, and listened. After I finished, he hitched up his pants with both thumbs, looked straight into my eyes, and in his most official voice replied: "All right, I'll look into it!" Shortly thereafter he created the parton model, and the following fall Feynman, with Finn Ravndal and Mark Kislinger, two graduate students, looked for evidence of concrete quarks in pion photoproduction, a place where I, and others, had never looked.

Three years later, as I was walking down the corridor on the fourth floor of Lauritsen where we both had offices, I noticed Feynman in the distance with an enormous grin, walking towards me like a sailor, rocking back and forth, thumbs hooked inside his belt, fingers spayed apart. When he was almost in my face, no more than a foot away, he extended his right hand and said "Congratulations Zweig! You got it right." By that time I had switched to neurobiology and didn't know that his work with Finn and Mark had finally been completed. 


\subsection{Bayes' theorem}

In retrospect, Bayes' theorem could have been used to elucidate some of the issues involved in deciding if aces should have been accepted as constituents of hadrons. With some massaging, Bayes' theorem says that the probability that hadrons have ace constituents, given the experimental evidence $(\phi \nrightarrow \rho+\pi$, mass relations, etc. $)$, is

$$
P(A \mid E)=\frac{1}{1+\lambda}, \text { where } \lambda=\frac{P(E \mid \bar{A})}{P(E \mid A)} \frac{P(\bar{A})}{P(A)} \approx \frac{P(E \mid \bar{A})}{P(A)},
$$

where $P(E \mid \bar{A})$ is the probability of the evidence given that aces do not exist, $P(A)$ is the a priori probability that they do exist, and $P(\bar{A})$ that they don't. The approximate form for $\lambda$ follows from

$$
P(\bar{A}) \approx P(E \mid A) \approx 1 .
$$

Whether or not a rational person would have believed in the existence of aces depends only on the ratio $\lambda$ of two small numbers. They would have believed if and only if the likelihood of obtaining the evidence, assuming that aces that didn't exist, was much lower than the a prior probability of aces existing i.e., if and only if

$$
P(E \mid \bar{A})<<P(A) .
$$

When this inequality holds, even though initially $P(\bar{A}) \approx 1$, when the experimental evidence is taken into account, just the opposite holds, i.e., $P(A \mid E) \approx 1$.

Since it is not possible to empirically determine these probabilities, how should this argument be interpreted? It suggests that in deciding whether hadrons have ace constituents, consider how easy it would be to find an alternative explanation for all the relations that follow assuming their existence, and weigh that against how much you like the idea of ace constituents in the abstract, without any experimental evidence for or against their existence. I suspect that those working in the RutherfordBohr tradition found acceptance easier than those trying to follow in Einstein's footsteps. ${ }^{22}$

\subsection{Different priors, different times}

The early adopters were Linus Pauling and Dick Dalitz, with Pauling coming first. Shortly after the CERN preprints were circulated, I received galley proofs of the third edition of Linus Pauling's "College Chemistry [13]," where Pauling presented aces to undergraduates. He asked for my comments and corrections. Essentially none were necessary. Pauling recognized a good thing when he saw it. Aces didn't have anything to do with college chemistry, but I suspect he thought aces were so beautiful, and so right, that he couldn't resist $(P(A)$ was not that small for him).

Dalitz's conversion is less surprising. He came from nuclear physics where nucleon constituents, and the forces between them, were his daily bread and butter. He just changed constituents.

For Feynman acceptance of concrete quarks came when he discovered them for himself in photoproduction amplitudes, where no one else had looked. Others were convinced by deep inelastic scattering experiments at SLAC. The holdouts finally folded with the discovery of the $\psi(\mathrm{J})$, whose forbidden decay modes and narrow width were just like $\phi$ with the fourth ace replacing $\Lambda_{0}$. It was "déjà vu all over again."

\footnotetext{
${ }^{22}$ A Baysian inspired evaluation of current speculative theories is also possible. If a theory has not yet advanced to the stage where it can compute observables, the evidence $E$ above, should be replaced by the Standard Model.
} 


\section{Invention or discovery}

Last summer Murray and I were present at a talk given by Chris Llewellyn Smith at the Santa Fe Institute. In his introduction, Geoffrey West said "Murray and George invented quarks, which were later discovered at SLAC." I interrupted to suggest a different ending to Geoffrey's sentence: "quarks, whose existence was later confirmed at SLAC." But what was invented, and what discovered? According to Merriam-Webster, discovery is "the act of finding or learning something for the first time," and invention is "a product of the imagination." Current quarks were invented in the tradition of Einstein. Aces were discovered in the Rutherford-Bohr tradition, buried in the data, obscured by the contradictions they implied. Bohr would have loved them.

\section{Final thoughts}

The CERN report concludes [19] :

"There are, however, many unanswered questions. Are aces particles? If so, what are their interactions? Do aces bind to form only deuces and treys? What is the particle (or particles) that is responsible for binding the aces? Why must one work with masses for the baryons and mass squares for the mesons? And more generally, why does so simple a model yield such a good approximation to nature?"

After dutifully listing possible interpretations of the results, the report concludes with:

"there is also the outside chance that the model is a closer approximation to nature than we may think, and that fractionally charged aces abound within us."

This then was the beginning of QCD, the theory that will eventually give us a real understanding of how it came to be that Becquerel's photographic plate clouded in his drawer.

\section{Epilogue}

Although Bohr was able to calculate the spectral lines of hydrogen, his model could not accurately account for the spectrum of helium, and was conceptually incomplete. For example, the wave nature of particles had not yet been discovered by de Broglie, and was absent from Bohr's thinking. There was no Lagrangian. There was no theory!

The concrete quark model provided predictions for the particle spectrum, and enabled calculations of hadronic masses and couplings that were accurate to varying degree, but it was conceptually incomplete, lacking a specification of the interaction between quarks. Like the wave-particle duality of quantum mechanics, concrete quarks were chimeric, sometimes acting as fields in a field theory for weak decay, sometimes as convenient objects for the calculation of masses and coupling constants. And like the Bohr atom, the $\Omega^{-}$, made from three quarks in the same state, could not exist according to the laws that were known. There was no Lagrangian. There was no theory!

Details aside, the important observation was that hadrons had fermion constituents of baryon number $1 / 3$, with dynamics that suggested they were real, and therefore should have corresponding fields. It was generally believed that hadrons had constituents, but those constituents were other hadrons. Some even thought that field theory was irrelevant. No! Concrete quarks said that there is a deeper level of reality to be described with field theory, and channelled thinking into more productive directions, leading to QCD. It was this deeper level of reality, with fractional charges, that made the acceptance of concrete quarks so difficult, and truly revolutionary. 
The regularities in the spectral lines of the hydrogen atom responsible for the creation of the Bohr model, that eventually led to the invention of quantum mechanics, were immediately derived from quantum mechanics. Although ever more accurate numerical calculations of masses of the low-lying hadrons are now possible with QCD, the regularities among these masses that led to the discovery of the concrete quark model have not been derived. Their derivation would provide a satisfying test of that fledgling theory.

How can such a complicated nonlinear theory as QCD give rise to the simple low-energy relations of the concrete quark model? There should be some way to approximate QCD so that the existence and hierarchy of relations among masses become apparent. It's not like the physics of water where the greater the nonlinearity, the more complex the flow. Quite the contrary. In QCD, when energies are small and nonlinearities large, the particle spectrum and its couplings are simple.

Recall the history of classical mechanics. Newton's laws were reformulated again and again over centuries, each time with a different purpose. In the following centuries QCD will be reformulated again and again, and with one of those reformulations the concrete quark model may appear, a fortiori.

\section{Acknowledgements}

I thank Erica Jen and Jeffrey Mandula for their insightful comments, and David Donoho, Stanislaw Mrowczynski, Finn Ravndal, and Harvey Shepard for improving the accuracy and content of the text.

\section{References}

[1] G. F. Chew and S. Frautschi, Phys. Rev. Letters 7, 394 (1961).

[2] G. F. Chew, M. Gell-Mann, and A.H. Rosenfeld, Scientific American, 74 (February 1964).

[3] P.L. Connolly, et al., Phys. Rev. Letters 10, 371 (1963).

[4] E. Fermi and C.N. Yang, Phys. Rev. 76, 1739 (1949).

[5] H. Geiger and E. Marsden, Proc. R. Soc. Lond. A 82 no. 557, 495 (1909).

[6] M. Gell-Mann and E.P. Rosenbaum, Scientific American, 72 (July 1957).

[7] M Gell-Mann, and A. H. Rosenfeld, Annu. Rev. Nucl. Sci. 7, 407 (1957).

[8] M. Gell-Mann, Phys. Lett., 8, 214 (1964).

[9] M. Gell-Mann, Acta Physica Austriaca, Suppl. IX, 733 (1972).

[10] S.J. Lindenbaum, Annu. Rev. Nucl. Sci. 7, 317 (1957).

[11] J. Mandula, J. Weyers, and G. Zweig, Annu. Rev. Nucl. Sci. 20, 289 (1970).

[12] S. Okubo, Phys. Lett. 5, 2, 165 (1963).

[13] L. Pauling, College Chemistry, 3rd ed., W. H. Freeman and Co., San Francisco (1964).

[14] M. Roos, Rev. Mod. Phys. 35, 314, (1963).

[15] S. Sakata, Progr. Theor. Phys. 16, 686 (1956).

[16] E. Rutherford, Phil. Mag. Series 6 21, 669 (1911).

[17] F. Zachariasen, Phys. Rev. Letters 7, 112 (1961); Erratum 7, 268 (1961).

[18] G. Zweig, Il Nuovo Cimento XXXII, no. 5, 689 (1964).

[19] G. Zweig, "An $\mathrm{SU}_{3}$ Model for Strong Interaction Symmetry and its Breaking," CERN Report 8419/TH.401 (January 17, 1964), http://cdsweb.cern.ch/record/352337?ln=en.

[20] G. Zweig, "An $\mathrm{SU}_{3}$ Model for Strong Interaction Symmetry and its Breaking II," CERN Report 8419/TH.412, (February 21, 1964), in Developments in the Quark Theory of Hadrons, A Reprint Collection, Volume I: 1964-1978, eds. D. B. Lichtenberg and S. P. Rosen, Hadronic Press, Inc., Nonantum, Mass., pp. 22-101 (1980), http://cdsweb.cern.ch/record/570209?ln=en. 
[21] G. Zweig, "Origins of the Quark Model," in Proceedings of the Fourth International Conference on Baryon Resonances, ed. N. Isgur, University of Toronto, Canada, 439-479, (1980), www-hep2.fzu.cz/ chyla/talks/others/zweig80.pdf.

[22] G. Zweig, "Memories of Murray and the Quark Model." Proceedings of the Conference in Honor of Murray Gell-Mann's 80th Birthday, eds. H. Fritzsch, K. K. Phua, B. E. Baaquie, World Scientific, Singapore, 7-20, (2010), http://arxiv.org/abs/1007.0494. 